\title{
¿Hacia dónde se encamina la epidemia de infección por VIH y sida?'
}

\author{
T. E. Mertens ${ }^{2}$ y D. Low-Beer ${ }^{3}$
}

RESUMEN En el transcurso del último decenio, muchos países en todo el mundo han establecido una vigilancia sistemática para casos de sida y de infección por virus de la inmunodeficiencia humana (VIH). Las estimaciones de la carga de infección por VIH que derivan de datos empíricos son indispensables para evaluar la situación en diferentes partes del mundo y las tendencias se emplean para seguir la trayectoria de epidemias regionales y así poder basar las actividades de intervención en hechos concretos. Se estimó a fines de 1995, a raíz de un extenso análisis de datos sobre la infección por VIH y el sida efectuado país por país, que ha habido un total acumulativo de 6 millones de casos de sida en adultos y niños en todo el mundo y que actualmente 20,1 millones de adultos están infectados por VIH o tienen sida. De la prevalencia total de infecciones por VIH, la mayor parte se concentra en África oriental, central y meridional, pero la epidemia está evolucionando y la infección se está propagando de las zonas urbanas a las rurales, y también al sur y occidente de África, a la India y al Asia Sudoriental y, en menor escala - con cambios similares en la proporción de infecciones heterosexuales-, a América del Norte, Europa occidental y América Latina.

Aunque no se puede confiablemente pronosticar en el largo plazo la magnitud de la epidemia de infección por VIH en el mundo, la proyección actual de la OMS es de un total acumulativo cercano a los 40 millones de infecciones por VIH en hombres, mujeres y niños para el año 2000. Llegado ese año, la razón de infecciones en hombres a infecciones en mujeres se aproximará a 1:1. Las tendencias más recientes indican que las tasas de prevalencia de VIH podrían estar en vías de estabilización o incluso de reducción en mujeres embarazadas en el sur del Zaire y en partes de Uganda, en reclutas militares de 21 años de edad en Tailandia y en algunas poblaciones del norte de Europa y de los Estados Unidos de América. Si bien estos cambios pueden ocurrir como parte de la dinámica intrínseca de la epidemia, hay indicios de que las reducciones de la prevalencia de la infección por VIH están relacionadas con disminuciones de la incidencia, que se deben, al menos en parte, a las actividades de prevención. El actual reto de los métodos de vigilancia y evaluación radica en descubrir aquellos ingredientes que han llevado al éxito y que pueden ofrecer un rayo de esperanza.

1 Se publicó en inglés en el Bulletin of the World Health Organization, 1996, Vol. 74, No. 2, con el título "HIV and AIDS: where is the epidemic going?". (C) Organización Mundial de la Salud, 1996.

2 Organización Mundial de la Salud, Ginebra, Suiza. Dirección postal: División de Desarrollo de Políticas, Programas y Evaluación, Organización Mundial de la Salud, 1211 Ginebra 27, Suiza. Las solicitudes de separatas (No. 5682) deben dirigirse a este autor.

3 St. John's College, Cambridge, Inglaterra. Exfuncionario (1993-1995) del Programa Mundial sobre el Sida, OMS, Ginebra, Suiza.
La epidemia de infección por virus de la inmunodeficiencia humana (VIH) y de sida, que ha surgido en los últimos 25 años del siglo $X X$, se ha diseminado en menos de dos decenios a más de 190 países en todos los continentes (1). La infección es causada por dos tipos principales de virus: VIH-1 y VIH-2. El VIH-1 causa la gran mayoría de las infecciones por VIH en el mundo; el VIH-2 parece estar bastante limitado al África occidental, con focos en Angola y Mozambique y unos cuantos casos en Europa, las Américas y la India (2). La infección por VIH se caracteriza, además, por una amplia diversidad genética de cepas víricas en diferentes partes del mundo (3). Varios estudios in vitro han mostrado importantes diferencias en las propiedades 
biológicas de los diferentes subtipos del VIH-1 (4), pero se sabe muy poco sobre sus posibles diferencias de transmisibilidad. En el presente documento, la abreviatura VIH se refiere al VIH-1.

En el último decenio, los programas nacionales sobre el sida, en colaboración con la OMS, han establecido actividades de vigilancia de sida y de infección por VIH en la mayor parte de los países. La vigilancia y las estimaciones de la carga de infección se pueden emplear sistemáticamente para evaluar la gravedad del problema, mantener focalizadas las actividades de intervención cuando se disponga de estimaciones por subpoblaciones, y seguir el desarrollo de las epidemias regionales en el marco de la situación mundial. En este trabajo se presentan los métodos de estimación y los resultados arrojados por las estimaciones mundiales de la infección por VIH y de casos de sida, se describen las características de la epidemia por subcontinentes y se discuten tendencias advertidas de importancia y sus posibles factores determinantes.

\section{MATERIALES Y MÉTODOS}

La OMS emplea varios métodos de estimación y pronóstico que se basan en datos empíricos derivados del sistema mundial de vigilancia epidemiológica de sida y de vigilancia centinela de la infección por VIH, y también en estudios publicados y los archivos nacionales de la OPS/OMS, actualizados continuamente por medio de contacto con los profesionales en cada país (5). El proceso se basa en una estrecha relación entre vigilancia, estimación, pronóstico y evaluación de tendencias, tanto para desarrollar la capacidad nacional como para asegurar que la interpretación de los resultados permita comparaciones dentro del contexto mundial. En otros trabajos se ha publicado un recuento detallado de estos métodos $(1,5)$. En breves palabras, la OMS basa sus estimaciones en un análisis de los estudios de seroprevalencia de VIH, los casos de sida noti- ficados, las estimaciones de subnotificación, el tamaño y la estructura de la población (incluso la composición por edad y sexo y las diferencias urbanas y rurales) y las formas de transmisión predominantes. Se combinan dos métodos importantes, el proceso de estimación de la infección por VIH y el uso de un método de pronóstico para fines de validación, con la coherencia entre la incidencia de VIH, la prevalencia y los casos de sida y sus proyecciones futuras.

El procedimiento mediante el cual se estima la prevalencia de infección en un país en particular puede describirse en cinco etapas básicas. Primero, se identifican subpoblaciones en las que se ha comprobado la presencia de infección por VIH mediante el análisis de todos los datos disponibles, independientemente de su calidad. Segundo, se analizan los estudios de prevalencia para determinar sus rasgos metodológicos, según los criterios descritos anteriormente (5), y se seleccionan los que tengan un tamaño muestral liminar previamente determinado. Tercero, se emplean todos los puntos restantes de los datos de seroprevalencia para establecer los límites máximos y mínimos para cada subpoblación y hacer una estimación conservadora (que, de ordinario, es inferior al valor mediano de todos los niveles de prevalencia), teniendo en cuenta las tendencias observadas en la seroprevalencia en los 2 años anteriores. Cuarto, se emplea la mejor información disponible para estimar el tamaño de la subpoblación. Por último, las tasas de prevalencia estimadas se aplican al tamaño estimado de las subpoblaciones y se suman para estimar la prevalencia de infección en el país (5).

Una vez que se ha obtenido una estimación provisional para un país en específico, se comparan los casos de sida notificados y estimados con el número que se podría esperar a la luz de los niveles estimados de infección por VIH pasados y presentes, aplicando un modelo de proyección (que suele ser, aunque no siempre, un modelo epidemiológico (6)) con los datos apropiados, y la estimación se ajusta según ello (1). Todas las estimaciones se discuten con la Oficina Regional de la OMS correspondiente y con el respectivo programa nacional sobre el sida.

Para estimar el número de futuras infecciones por VIH y de casos de sida actuales y futuros en el corto plazo, la OMS emplea un modelo basado en la historia natural del VIH y una curva teórica de la epidemia, en forma de gamma (6). Esto exige el manejo de tres datos: la prevalencia actual estimada de las infecciones por VIH, el año en que empezó la epidemia y la actual posición en que esta se sitúa a lo largo de la curva epidémica. La forma y la posición en la curva epidémica cambian según los datos arrojados por las actividades de vigilancia, particularmente la trayectoria de los casos de sida a lo largo del tiempo. Partiendo de ahí, el modelo calcula el número total de infecciones desde el comienzo de la epidemia, lo cual es necesario para determinar la prevalencia actual de infección por VIH. El número total de infecciones se distribuye a lo largo de la curva gamma de incidencia de VIH para producir el número anual de infecciones nuevas por VIH. Más tarde la distribución de los datos de incubación se aplica a esas infecciones anuales para determinar el número anual de casos de sida.

Las proyecciones a corto plazo de las futuras infecciones por VIH se producen haciendo una extrapolación a lo largo de la curva epidémica más allá de la posición actual para derivar el número de infecciones nuevas que habrá en años futuros. La distribución de los datos de incubación se aplica a esas nuevas infecciones con el fin de producir estimaciones del número de casos de sida en el futuro.

\section{RESULTADOS}

\section{Estimaciones mundiales}

El 15 de diciembre de 1995, ya se habían notificado a la OMS 1291810 casos de sida en total (7), pero esta organización calcula que para fines de 
FIGURA 1. Distribución mundial estimada de la prevalencia de VIH y sida en adultos, por continente o región, a fines de 1995

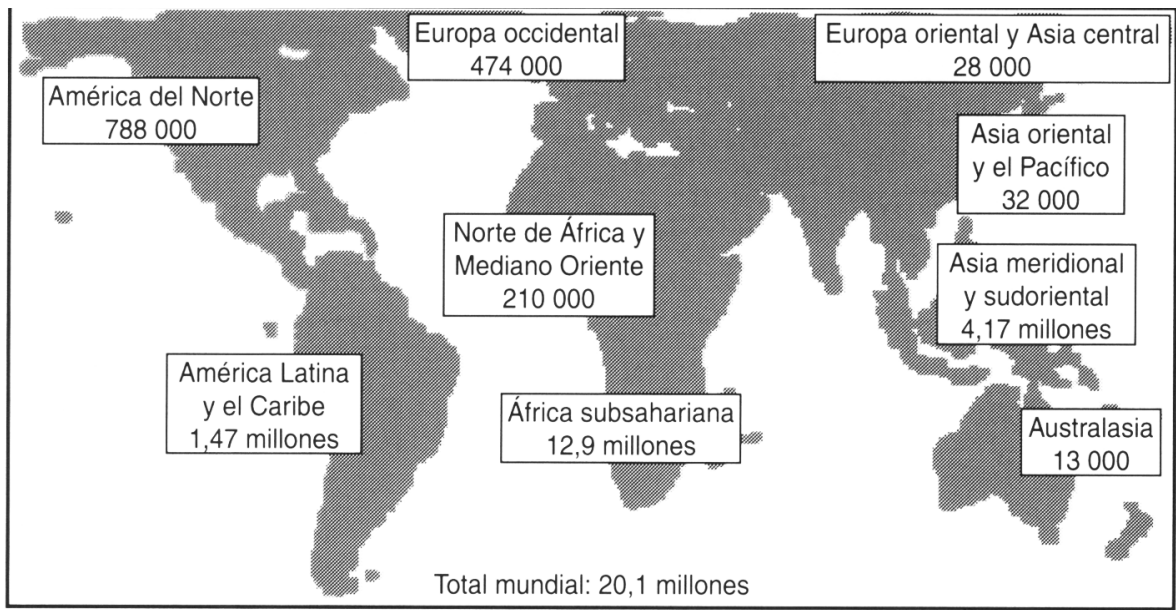

ese mismo año, si se tienen en cuenta el subdiagnóstico, la subnotificación y las demoras en la notificación, así como el número estimado de infecciones por VIH, el total acumulativo de casos de sida en el mundo era de 6 millones. Se estima que de ellos, casi 1,5 millones eran casos pediátricos de sida producidos por la transmisión del VIH de madre a hijo, principalmente en África subsahariana.

La OMS ha estimado, después de fines de 1995 y tras un análisis de datos sobre VIH y sida efectuado país por país (7), que desde que los VIH empezaron a propagarse por América del Norte, Europa y África subsahariana, alrededor de 24 millones de adultos han contraído la infección (7). La figura 1 muestra la distribución mundial de la prevalencia de infección por VIH en adultos, en la que se incluye a las personas vivientes que padecen de sida. A medida que van muriendo de sida las personas que estaban infectadas por VIH cuando comenzaba la epidemia, se va ampliando la brecha entre la prevalencia e incidencia acumulativa de infección por VIH.

En el mundo hay actualmente tres hombres infectados por cada dos mujeres y en el año 2000 el número de infecciones nuevas en mujeres se aproximará más al número en hombres (1). La figura 2 muestra las tendencias observadas en algunos países en la proporción de casos de sida en mujeres. En África subsahariana, la razón de hombres a mujeres es cercana a 1:1, mientras que en el resto del mundo se observa un aumento continuo de los casos de sida en mujeres. ${ }^{4}$ Las crecientes tasas de infección en la población femenina se acompañan de un aumento correspondiente del número de niños que nacen con la infección por VIH. Se estima que hasta la fecha más de 1,5 millones de niños han contraído la infección por VIH debido a la transmisión de madre a hijo (1). Estos niños pronto se enferman de sida $y$ mueren, por lo general antes de los 5 años, aunque en los Estados Unidos y Europa el período de incubación puede ser más largo.

Estas cifras son el testimonio de la tragedia que se ha venido desencadenando en los últimos 15 años y apuntan a la rápida propagación de $\mathrm{VIH}$ que aún se observa en todos los continentes. Actualmente hay indicios, sin embargo, de que se está produciendo una estabilización y hasta posiblemente una reducción de la prevalencia de la infección por VIH en algunas zonas y poblaciones de las regiones

\footnotetext{
4 Low-Beer D, et al. The global burden of HIV. (Datos inéditos).
}

FIGURA 2. Tendencias observadas en el porcentaje de casos de sida notificados en mujeres en determinados países o zonas, 1985-1993

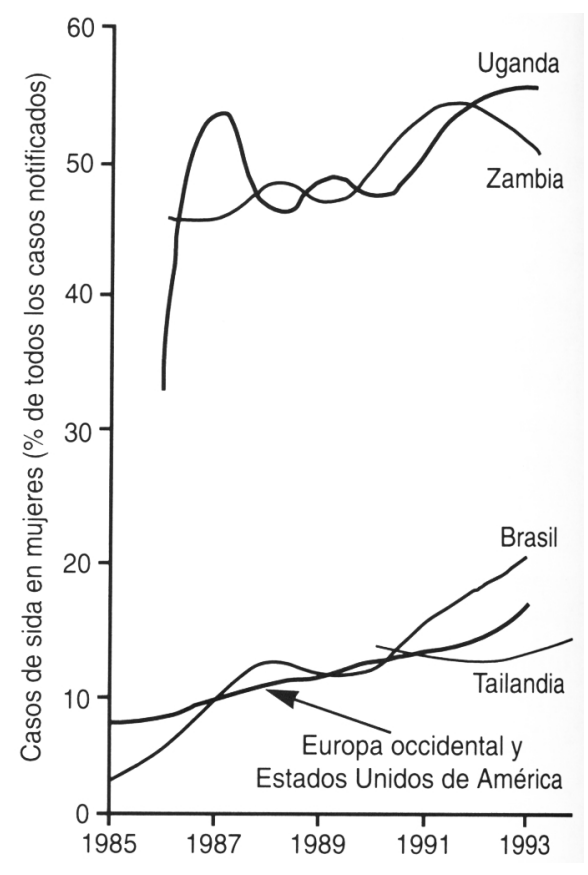

industrializadas de Australasia, América del Norte y Europa occidental, así como en las zonas de alta prevalencia de África oriental y central y en algunas poblaciones de Tailandia $(1,5)$.

La transmisión de VIH de madre a hijo incluye su transmisión durante el embarazo o parto y durante la lactancia natural. Puede que la amplia variación de las tasas de transmisión se deba a diferencias en la distribución de los factores que la determinan, como el grado de inmunodeficiencia de la madre, la presencia de corioamnionitis, la carencia materna de vitamina A o la exposición a leche materna infectada (8). En una población en que no se acostumbra amamantar a los niños, la mayoría de los casos de transmisión de madre a hijo se producen durante el embarazo y el parto, aunque según datos obtenidos de varios países previos a 1992, hasta 15\% de los bebés amamantados por madres infectadas por VIH pueden infectarse durante la lactancia natural (9). En lo que respecta a la transmisión heterosexual, las tasas de transmisión de madre a hijo 
se ven fuertemente influenciadas por el grado de viremia de las madres, habiéndose observado que mientras más alta la viremia, mayor es la infectividad (8).

Las estimaciones mundiales de las infecciones por VIH representan solo parcialmente el efecto de la epidemia en otras enfermedades. Por ejemplo, un aumento alarmante del número de casos de tuberculosis, paralelo a la epidemia de sida, se ha notificado en muchos países, particularmente en el África (10). La tuberculosis ya es una de las principales causas de defunción en adultos de países en desarrollo, donde cobra la vida de unos 3 millones de personas al año. Actualmente, la infección por VIH es uno de los principales factores de riesgo conocidos de tuberculosis activa en países en desarrollo cuya prevalencia de infección por VIH es alta, debido básicamente a la inmunodeficiencia que provoca. La aparición y propagación continua de la infección por VIH complican de forma apreciable los programas de lucha antituberculosa en países o zonas muy afectados por la epidemia de sida.

\section{Las epidemias subcontinentales}

Una característica distintiva de la epidemia, que hace 15 años comenzó con la detección de focos limitados de casos de sida, ha sido su capacidad para adquirir proporciones mundiales. Sin embargo, la propagación de VIH muestra distintas características en diferentes sociedades y una de las metas de la vigilancia mundial es proporcionar un marco que permita identificar y comparar los patrones de propagación en distintas regiones.

Europa occidental, América del Norte, Australasia. En esta región considerada como un todo, se había presentado, a fines de 1995, un total acumulativo de más de 1,8 millones de infecciones por VIH en adultos, de las cuales 1,2 millones se produjeron en los Estados Unidos. En total, la OMS estima que desde entonces ha habido más de 700000 casos acumulativos de sida en adultos solamente.

El VIH ha afectado principalmente a hombres homosexuales y a usuarios de drogas inyectables (1), así como a las parejas sexuales de estos individuos. La proporción de infecciones adquiridas por contacto heterosexual ha aumentado a paso lento en el último decenio y su incremento ha sido particularmente notable en poblaciones urbanas con tasas elevadas de uso de drogas inyectables (1), de crack (sustancia a base de cocaína) en el caso de Estados Unidos, o enfermedades de transmisión sexual (ETS) (10). Son marcadas las diferencias entre países, y aun dentro de un mismo país, en lo referente a la distribución de casos de sida en hombres homosexuales y usuarios de drogas inyectables, lo cual refleja la variabilidad de los patrones de transmisión de VIH. En Europa, por ejemplo, la mayor parte de los casos de sida en Escandinavia e Inglaterra han ocurrido en hombres homosexuales, mientras que dos tercios o más de los casos de sida notificados en Italia y España han sido en usuarios de drogas inyectables (11).

América Latina y el Caribe. La OMS estimó, a fines de 1995, que había un total acumulativo de más de 1,7 millones de infecciones por $\mathrm{VIH}$ en adultos y una prevalencia de unos 1,5 millones de casos (figura 1) (1). En ese momento, más de 140000 casos de sida en adultos y niños ya habían sido notificados (9). Tanto el número absoluto como la tasa de casos de sida por número de habitantes son mayores en el Brasil, donde más de 70000 casos se habían notificado a fines de 1995.

En América Latina, la mayor parte de las infecciones que ocurrieron en un principio fueron en hombres homosexuales o bisexuales. No obstante, desde fines de los años ochenta se ha producido un aumento de la transmisión heterosexual, sobre todo en hombres bisexuales y en sus parejas sexuales femeninas, así como en trabajadoras sexuales y sus clientes. Sirven de ejemplo datos del Brasil sobre hom- bres tratados en dispensarios de ETS, según los cuales hay una extensa distribución geográfica de VIH en todo el país (1). Las infecciones por VIH en usuarios de drogas inyectables constituyen, asimismo, un problema creciente en todo el subcontinente: por ejemplo, la prevalencia de infección por VIH en usuarios de drogas inyectables oscila entre 30 y 50\% en la Argentina y entre 20 y $60 \%$ en el Brasil (1).

En casi todo el Caribe, la transmisión heterosexual ha sido la vía de transmisión predominante por lo menos durante un decenio (1). Estudios efectuados en el contexto de programas nacionales para embarazadas tratadas en 1990 y 1991 en dispensarios de atención prenatal revelaron tasas de prevalencia de VIH cercanas a $3 \%$ en las Bahamas y de más de $1 \%$ en Santo Domingo (República Dominicana). En Haití, la prevalencia de VIH en mujeres atendidas en dispensarios de atención prenatal en 1993 fue de 7,5\% en zonas urbanas y de $5,5 \%$ en zonas rurales, y la tasa de seropositividad más alta se registró en los grupos más jóvenes (1).

En esta región la epidemia es muy heterogénea. La vigilancia es particularmente importante para monitorear las tendencias observadas en la proporción de la epidemia atribuible a casos heterosexuales, a fin de responder con estrategias de prevención apropiadas.

África subsahariana. La OMS estimó, a fines de 1995, que había 4,5 millones de casos de sida en adultos y niños y 16 millones de infecciones acumulativas por VIH en adultos, de los cuales todavía están vivos 12,9 millones (incluidas las personas con sida) (figura 1). Según pruebas epidemiológicas, el coito heterosexual es, con mucho, la forma de transmisión más común en esta región.

África oriental y central tienen de 50 a $60 \%$ de todas las infecciones por VIH que han surgido en África subsahariana, pero solo $15 \%$ de la población total de la región (1). En los últimos años se han acopiado datos sobre la 
incidencia de infección por VIH. Estos datos son importantes porque dicha incidencia determina de manera directa los patrones de infección y el futuro curso de la epidemia. En algunas partes rurales del sur de Uganda las tasas de seroprevalencia se han estimado en más de $10 \%$ de la población total (OMS, datos inéditos), pero según un estudio reciente, en el distrito rural de Massaka, situado en el sudoeste de
Uganda, las tasas de incidencia anual en adultos (mayores de 12 años) se redujeron de $7,5 \%$ en el período de 1989 a 1990, a 4,6\% en 1993 (12).

En África occidental y meridional, la epidemia sigue evolucionando. En 1992, la prevalencia de VIH en mujeres tratadas en dispensarios de atención prenatal en un estado de Nigeria había llegado a 6\% (1) y actualmente están apareciendo tasas similares de preva- lencia de VIH en África meridional. Dos encuestas transversales en serie, realizadas en marzo de 1992 y diciembre de 1993 en mujeres tratadas en dispensarios de atención prenatal en un distrito rural del nordeste de Sudáfrica, mostraron un aumento de la seroprevalencia de 4,2 a 7,9\% (1). La figura 3 muestra las tendencias observadas en Sudáfrica de 1991 a 1994 en la prevalencia de infección por VIH en mujeres

FIGURA 3. Izquierda. Tendencias de la prevalencia de VIH, según el resultado de la vigilancia centinela en determinados grupos en Sudáfrica, 1991-1994. Derecha. Tendencias de la prevalencia de VIH, según el resultado de actividades de vigilancia centinela en determinados grupos y ciudades de la India, 1987-1993
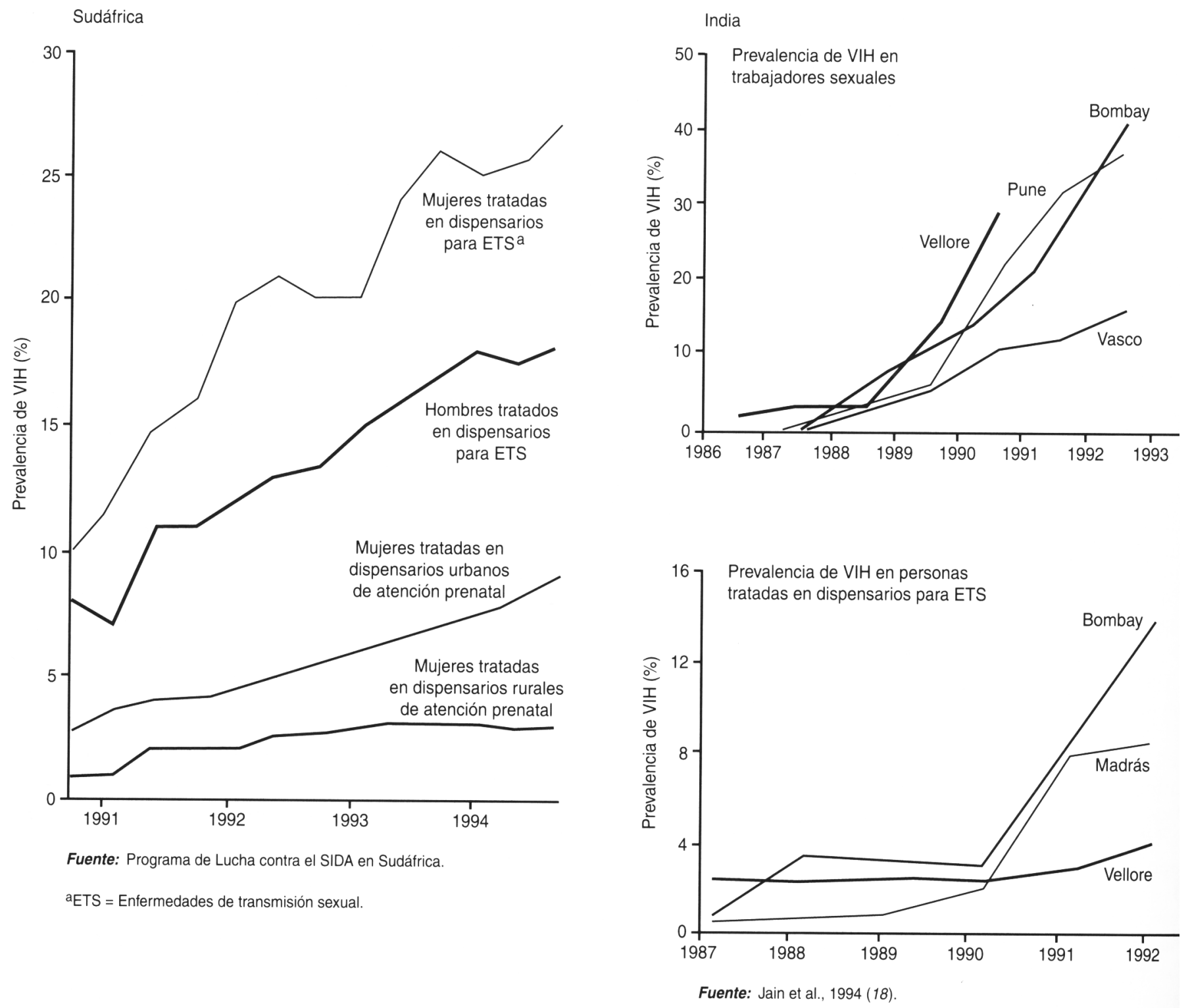
tratadas en dispensarios de atención prenatal y en pacientes de ETS.

No obstante el sombrío panorama que existe en gran parte del territorio de África subsahariana, hay indicios de que en ciertos países la prevalencia de VIH sigue siendo relativamente baja en la población en general (10). Por ejemplo, en Camerún y Benin, las tasas de prevalencia en mujeres tratadas en dispensarios de atención prenatal parecen haber sido menores de 5\% en el período de 1989 a 1993 (OMS, datos inéditos). Las razones de las disparidades en la propagación de la epidemia a lo largo del subcontinente deben explorarse mediante un examen estandarizado de los patrones de apareamiento sexual en varias partes del mundo. También se necesitan investigaciones adicionales destinadas a evaluar cualquier contribución de la variabilidad genética del VIH a la infectividad y transmisión.

Asia meridional y sudoriental. En esta región, la progresión de la epidemia ha sido rápida en varias poblaciones desde fines de los años ochenta. A fines de 1995, la OMS estimó que había más de 4,25 millones de infecciones acumulativas por VIH en adultos (figura 1). Se cree que a fines de 1995 ya habían ocurrido alrededor de 300000 casos acumulativos de sida, pero hasta entonces solo 25090 de ellos se habían notificado.

En Tailandia, los VIH se propagaron primero entre usuarios de drogas inyectables, y posteriormente entre trabajadores sexuales comerciales y sus clientes, y de las parejas regulares de estos clientes a la población en general. Hace poco se ha notificado que en usuarios de drogas inyectables en Bangkok las tasas de seroconversión aumentaron aceleradamente a fines de los años ochenta, y sufrieron un relativo descenso y estabilización alrededor de 1991-1992 (13). Los datos del programa nacional sobre el sida indican que la prevalencia actual de VIH en mujeres tratadas en dispensarios de atención prenatal es de $8 \%$ en el norte del país, mientras que la prevalencia mediana en todo el país era de
1,7\% a mediados de 1994 (1). La prevalencia en trabajadoras sexuales del norte de Tailandia era de mucho más de $30 \%$, mientras que en reclutas militares la prevalencia fue mayor de $7 \%$ en 1993 (OMS, datos inéditos). Sin embargo, la incidencia parece haberse reducido recientemente en algunas trabajadoras sexuales (1) y en reclutas del Real Ejército de Tailandia en el norte del país, grupos en que se observó una incidencia de 0,87 por 100 años-persona en comparación con tasas de incidencia previas de alrededor de 3,4\% al año. Esto se refleja, en parte, en una reducción más reciente de la prevalencia de VIH (figura 4).

En la India, que tiene $16 \%$ de la población mundial, se ha comprobado infección por VIH en usuarios de drogas inyectables, en trabajadores sexuales de uno y otro sexo y en la población de ciertos centros urbanos (1). Es difícil tener una idea precisa de la propagación de VIH en diferentes partes de la India debido a la variabilidad de entornos epidemiológicos, a la enorme cantidad de zonas urbanas y semiurbanas y a la falta de uniformidad de las encuestas serológicas en lo que respecta al período de estudio y a la población analizada. A pesar de estos problemas, una actividad concertada de vigilancia ha generado una mayor disponibilidad de datos sobre los gra- dos de infección por VIH y sus tendencias en subpoblaciones y estos datos sirven para orientar las intervenciones y los pronósticos. En Madrás, al sur, la seroprevalencia en pacientes con ETS aumentó de cerca de $1 \%$ en el período de 1986 a 1989 a $8,5 \%$ en el de 1991 a 1992 (1). En el Estado de Maharashtra al occidente, que tiene cerca de 100 millones de habitantes y una población urbana de más de 23 millones, la epidemia ya está bien establecida: los resultados de la primera ronda de vigilancia centinela realizada en 1994 entre mujeres tratadas en dispensarios de atención prenatal en Bombay indicaron una prevalencia de 2,5\% (1). La figura 3 muestra los patrones de infección por VIH en ciertas poblaciones de diferentes ciudades de la India. Según los datos disponibles, la epidemia aún no ha empezado a propagarse en el norte, centro y oriente del país, aunque la escasez de encuestas serológicas rigurosas impide llegar a cualquier conclusión (1).

También se ha visto una rápida propagación de VIH a la población en general en algunas partes urbanas y rurales de Myanmar, contiguas a Tailandia. En Camboya, la epidemia se ha propagado por contacto heterosexual, y a mediados de 1994 las tasas de prevalencia entre donantes de sangre en Phnom Penh eran de 3,5\% (1). En Viet

\section{FIGURA 4. Tendencias observadas en la prevalencia de VIH en reclutas del ejército de Tailandia, 1989-1994}

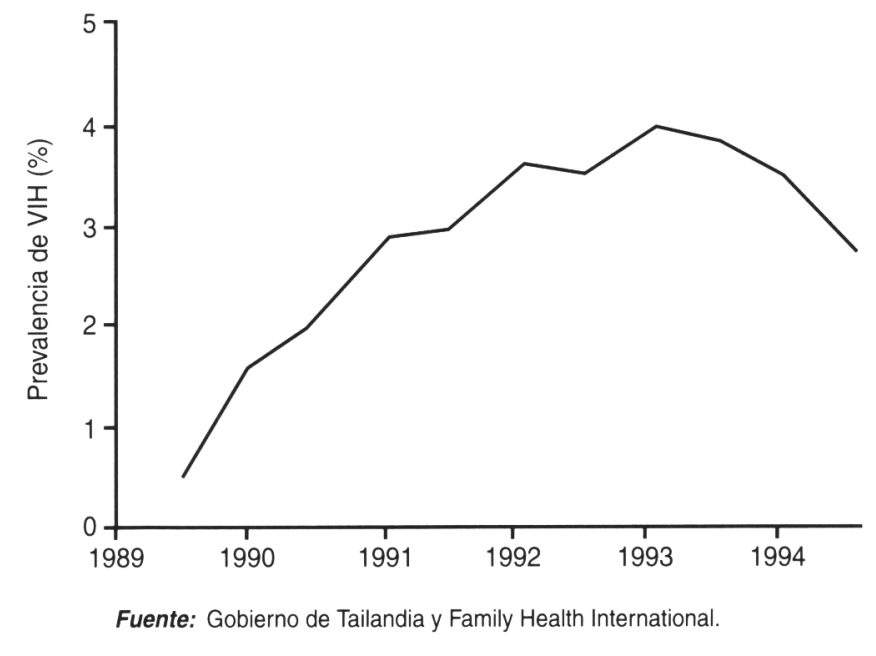


Nam se observó un aumento de la seroprevalencia de 2 a $30 \%$ entre los usuarios de drogas inyectables en el período de 1992 a 1993 (5). El grado de propagación de la epidemia está menos definido en Bangladesh, Indonesia y el Pakistán.

Región del Pacífico occidental, Europa oriental y Asia central. La OMS ha estimado que a fines de 1995 se habían producido en la región del Pacífico occidental 32000 infecciones por VIH en adultos. También en este caso se observan diversas vías de transmisión en distintas partes de la región. Una gran proporción de los casos de sida notificados en el Japón corresponde a hemofílicos que recibieron transfusiones de hemoderivados infectados por VIH entre comienzos y mediados de los años ochenta. Si la epidemia se llegara a afianzar en la China, la posibilidad de un gran aumento del número de infecciones por VIH en el país más populoso del mundo, aun siendo bajas las tasas de incidencia, sería muy alarmante (1).

La magnitud del problema del VIH y sida en Europa oriental y Asia central sigue siendo ambigua. En San Petersburgo, Rusia, la mayor parte de las infecciones más recientes se atribuyen a transmisión heterosexual (1). En algunos países de Europa oriental el uso de drogas inyectables se asocia con tasas muy altas de infección por VIH: un estudio transversal realizado en Varsovia, Polonia, en 1993 mostró una seroprevalencia de $46 \%$ entre los usuarios de drogas inyectables atendidos en los centros de tratamiento (1). En gran parte de la región se están produciendo cambios sociales y estructurales que probablemente hagan aumentar el riesgo de transmisión de VIH.

Norte de África y el Mediano Oriente. A fines de 1995, la OMS estimó una prevalencia de alrededor de 210000 infecciones por VIH en adultos en la región, que comprende el Sudán, territorio contiguo a la parte oriental del África subsahariana, pero el grado de propagación no se sabe con seguridad y a fines de 1995 solo se habían notificado 3474 casos de sida. Esta incertidumbre se debe en parte a grandes brechas en la vigilancia de VIH, y la mayor parte de los datos disponibles provienen del Norte de África. En Marruecos, la prevalencia de infección por VIH en donantes de sangre fue de 0,01\% en 1993 (1). En Nouakchott, Mauritania, la prevalencia en este grupo durante el período de 1988 a 1992 fue de 0,4\% (1). En Eritrea, los datos obtenidos por medio de una serovigilancia nacional efectuada en 1994 indicaron una tasa de prevalencia de $25 \%$ en trabajadores sexuales, $2,7 \%$ en donantes de sangre y $3 \%$ en mujeres tratadas en dispensarios de atención prenatal (1).

Proyecciones mundiales de la infección por VIH y sida. Como resultado de las tasas de infección por VIH, se espera que el número de casos de sida nuevos se estabilice en gran parte del mundo industrializado a mediados de los años noventa y que siga aumentando en la mayor parte del mundo en desarrollo, donde la infraestructura de atención de salud ya está sobrecargada por otras enfermedades. Se pronostica que para el año 2000 alrededor de 700000 y 500000 casos nuevos de sida se producirán cada año en África y Asia, respectivamente.

No se puede pronosticar confiablemente la magnitud de la pandemia de infección por VIH y sida en el plazo más largo. Basándose, no obstante, en los datos disponibles sobre la situación actual de la pandemia en el mundo y sus recientes patrones de propagación, la OMS ha generado una serie de proyecciones verosímiles. Para el año 2000, la OMS pronostica actualmente un total acumulativo de alrededor de 40 millones de infecciones por VIH en hombres, mujeres y niños, de las cuales más de $90 \%$ ocurrirán en países en desarrollo. El total acumulativo de casos de sida en adultos que actualmente se proyecta para el año 2000 es de mucho más de 10 millones. En cifras acumulativas, para el año 2000 hasta 5 millones de niños menores de 5 años habrán adquirido de la madre la infección por VIH, principalmente en África subsahariana.

Las proyecciones del número de casos de sida en lactantes y niños pequeños se basan en tasas de transmisión perinatal de alrededor de $30 \%$ y en tasas de fecundidad específicas. Debido, sin embargo, a que las madres infectadas probablemente mueran de sida en un período de 5 a 10 años después de dar a luz, los lactantes no infectados constituirán una población de huérfanos cada vez mayor. La OMS estima que cerca de 5 millones de niños menores de 10 años habrán quedado huérfanos para fines de los años noventa como resultado de la muerte de la madre debido a la infección por VIH. El número de huérfanos de madre -y también de padreaumentará aun más en los primeros años del siglo venidero como consecuencia de la muerte de padres y madres que quedaron infectados por VIH en los años noventa.

\section{DISCUSIÓN}

De toda la prevalencia de infección por VIH, la mayor parte sigue concentrada en el África oriental, central y meridional, pero la epidemia sigue evolucionando, con propagación de la infección del medio urbano al medio rural, así como a las partes sur y occidental del África, a la India y Asia sudoriental y, en menor grado, y con cambios de similares proporciones a infecciones heterosexuales, en América del Norte, Europa occidental y América Latina.

La pasada concentración de infecciones ha llevado a un inevitable aumento del número de casos de sida y de las tasas de mortalidad posteriores. Según datos recientes, en gran parte del mundo desarrollado y en desarrollo el sida se está convirtiendo en la causa más importante de defunción en adultos de 25 a 44 años. A pesar de que solo se han identificado de 55 a $85 \%$ de las defunciones relacionadas con $\mathrm{VIH}$, en 66 ciudades de los Estados Unidos el 
sida es la principal causa de defunción en hombres de 25 a 44 años (14). En algunos países de África que están más adentrados en la epidemia, más de la mitad de la mortalidad en adultos se atribuye actualmente a la infección por VIH (15). En Zambia, la tasa bruta de mortalidad en empleados de 33 fábricas y otros negocios aumentó precipitadamente de 2,5 por 1000 en 1987 a 183 en 1993, incremento que se atribuyó casi por completo a casos de enfermedad por VIH (16).

Las tasas de prevalencia de VIH en epidemias de un mínimo de 10 años se han estabilizado y ya han bajado en poblaciones de homosexuales en Europa y Estados Unidos. En este último país se observó una tendencia descendente a lo largo del tiempo entre homosexuales, en correlación con una reducción de otras enfermedades de transmisión sexual, cambios de comportamiento e intervenciones de salud pública. También hay datos fidedignos que sugieren una estabilización o un descenso de la prevalencia de VIH en mujeres embarazadas en el sur del Zaire desde 1989, así como en la población de algunos distritos rurales (12) y en zonas urbanas de Uganda (OMS, datos inéditos). Estas tendencias también se han observado al analizar los datos nacionales generados por la vigilancia de casos de sida en este último país (OMS, datos inéditos). Se han observado cambios cualitativos de importancia en la epidemia, de los cuales son ejemplos una mayor tendencia hacia la transmisión heterosexual y hacia una proporción más grande de infecciones por VIH en grupos más jóvenes. Estos cambios deben entenderse a la luz de los distintos patrones de incidencia subyacente de VIH que corresponden a los diferentes grupos de edad y de población (1).

Tanto los datos como los modelos estadísticos revelan que la epidemia se encuentra en una etapa de crecimiento más rápido y limitado en sentido temporal que la descrita previamente (1, $10,17)$ y en algunas subpoblaciones la explosión inicial de la epidemia puede tardar de 1 a 4 años. Por ejemplo, la prevalencia de VIH en usuarios de drogas inyectables en Tailandia aumentó de 1\% en 1986 hasta que se estabilizó en $43 \%$ en 1988 y posteriormente la incidencia de infección por VIH se redujo y se volvió a estabilizar hasta quedar en un nivel muy inferior al de la incidencia máxima (13). La vigilancia mundial continua y sistemática de VIH demostrará si esos indicadores del curso que está siguiendo la epidemia se duplican en el contexto más general constituido por diferentes poblaciones de África, Asia y América Latina.

La diversidad de tendencias que está poniendo de manifiesto la vigilancia mundial plantea dos interrogantes difíciles de contestar. Primero, ¿qué tendencias subyacentes de la incidencia de VIH determinan los patrones de prevalencia de infección por VIH y sida? Es fundamental describir y explicar la curva de incidencia de VIH para poder hacer estimaciones, pronósticos e intervenciones eficaces. Por ejemplo, la reducción de la incidencia, junto con un aumento de la mortalidad, produce una tendencia descendente de la prevalencia de VIH. En la población en general, los cambios en las tasas de incidencia de VIH a lo largo del tiempo, que, sumados a los cambios de la mortalidad relacionada con el sida, dan origen a las fluctuaciones de las tasas de prevalencia de infección por VIH, pueden tener su explicación en una serie de factores. Entre estos cabe citar la saturación, así como posibles cambios de comportamiento, alteraciones potenciales de la virulencia del VIH-1 o de la inmunidad natural, o una modificación a lo largo del tiempo de la infectividad de la población con VIH. El grado de viremia es alto inmediatamente después de contraer la infección por estos virus y permanece así por un período de varias semanas. Más tarde se reduce, estabilizándose por muchos años antes de elevarse nuevamente cuando se presentan las enfermedades relacionadas con VIH. Esta evolución temporal de la viremia se describe como "efecto de bañera" y parece estar relacionada con la eficacia de la transmisión de VIH en el sentido de que mientras mayores son los nive- les, más alta es la infectividad $(1,5,10$, 17). Esto último puede tener amplias implicaciones en lo que respecta a la dinámica de la epidemia en diferentes países. Si se toma a una población que mantiene un mismo comportamiento sexual y los mismos patrones de apareamiento a lo largo del tiempo, las variaciones de la infectividad pueden ser la fuerza que da impulso a la rápida fase de expansión, y es posible que ayude a reducir la incidencia general de VIH en una etapa más tardía de la trayectoria de una epidemia dada, a medida que disminuye la eficacia general de la transmisión. En la fase temprana de cualquier epidemia, el número de personas sumamente infecciosas es mayor que en etapas más avanzadas (1). Es probable, sin embargo, que una combinación de varios de los cuatro factores citados determine los cambios en la incidencia de VIH.

Pese a que la estabilización o reducción de la prevalencia de VIH puede ocurrir independientemente de las actividades de prevención y como resultado de la dinámica intrínseca de la epidemia, es factible que la reducción de la incidencia observada en trabajadores sexuales y reclutas del Real Ejército de Tailandia en el norte del país o en partes de África oriental se deban genuinamente a las actividades de prevención. Puede que estas reducciones reflejen una disminución del número de contactos sexuales comerciales, o bien el éxito de la política establecida por el Gobierno de Tailandia de usar condones obligatoriamente en los prostíbulos, el efecto que el mejor control de las ETS ha tenido en la incidencia de infección por VIH (18), o cambios de comportamiento tras haber presenciado la muerte de muchos parientes.

El segundo interrogante es cómo se puede evaluar el efecto de las intervenciones y de otros factores que determinan los diversos patrones de infección revelados por la vigilancia mundial. Se ha observado una disminución del número de casos de ETS en comunidades donde las intervenciones se han llevado a cabo exitosamente 
$y$, en el transcurso de este último año, en todo el territorio de Tailandia (1). El poder demostrar que las intervenciones de salud pública influyen en el curso general de la epidemia dependerá en gran medida de la duplicación de los datos revelados por la vigilancia en poblaciones más generales de África, Asia y América Latina y de una minuciosa evaluación. La OMS ha preparado un conjunto de medidas de evaluación $(19,20)$ y ha puesto en marcha actividades de evaluación estrechamente vinculadas con los adelantos de los últimos 10 años en la vigilancia de VIH y sida. Es indispensable mantener la continuidad dentro del marco de la vigilancia mundial a lo largo del tiempo, para poder interpretar aquellas tendencias originadas por las actividades de intervención que comienzan a influir en la epidemia y a dar un rayo de esperanza.

Agradecimiento. Expresamos nuestro agradecimiento a los programas nacionales sobre el sida en todo el mundo, ya que sin su sostenida actividad de acopio de datos y su confianza en la OMS (tanto en las Oficinas Regionales como en la sede) sería imposible realizar la vigilancia mundial de la pandemia de VIH y sida; al Centro Europeo de Vigilancia Epidemiológica del Sida (uno de los Centros Colaboradores de la OMS) por su apoyo con la observación de las tendencias en Europa; a la Oficina del Censo de los Estados Unidos por la prontitud con que suministró información sobre los estudios publicados al Programa Mundial sobre el Sida (así como a los autores de esos estudios) y a A. Burton, R. Stoneburner y P. Sato por su asistencia.

\section{REFERENCIAS}

1. Mertens TE, et al. Global estimates of HIV infections and AIDS: further heterogeneity in spread and impact. AIDS 1995;9(supl 1): S251-S272.

2. De Cock KM, et al. Epidemiology of HIV-2. Why there is no HIV-2 pandemic. JAMA 1993; 270:2083-2086.

3. Sharp PM, et al. Origins and diversity of human immunodeficiency viruses. AIDS 1994;8(supl 1):S27-S42.

4. Kuiken CL, Korber BTM. Epidemiological significance of intra- and inter-person variation of HIV-1. AIDS 1994;8(supl 1):S73-S83.

5. Mertens TE, et al. Global estimates and epidemiology of HIV infections and AIDS. AIDS 1994;8(supl):S361-S372.

6. Chin J, Lwanga K. Estimation and projection of adult AIDS cases: a simple epidemiological model. Bull World Health Organ 1991;69: 399-406.

7. Acquired immunodeficiency syndrome (AIDS) - data as of 15 December 1995. Provisional working estimates of adult HIV prevalence as of end 1994, by country. Wkly Epidemiol Rec 1995;70:353-357.
8. Van de Perre $\mathrm{P}$, Meda N. Interventions to reduce mother to child transmission of HIV. AIDS 1995;9(supl A):S59-S66.

9. Dunn DT, et al. Risk of human immunodeficiency virus 1 transmission through breastfeeding. Lancet 1992;340:585-588.

10. Mertens TE, Piot P. The epidemiology of HIV/AIDS: general considerations. En: De Vita VT, et al., eds. AIDS: etiology, diagnosis, treatment, and prevention. Philadelphia: Lippincott; 1996.

11. European Center for the Epidemiological Monitoring of AIDS. AIDS surveillance in the European Community and C.O.S.T. countries. Paris: Hôpital national de St. Maurice; 1994. (Informe 27).

12. Kengeya-Kayondo J, et al. HIV-1 incidence in adults and risk factors for seroconversion in a rural population in Uganda: 3 years of followup. En: Proceedings of the 10th International Conference on AIDS. Yokohama; 1994. (Resumen 068C).

13. Kitayaporn D, et al. HIV-1 incidence determined retrospectively among drug users in Bangkok, Thailand. AIDS 1994;8:1443-1450.
14. Selik RM, Chu SY, Buehler JW. HIV infection as leading cause of death among young adults in US cities and states. JAMA 1993;269: 2991-2994

15. Mulder DW, et al. Two-year HIV-1 associated mortality in a Ugandan rural population. Lancet 1994;343:1021-1023.

16. Baggaley, et al. Impact of HIV infection on Zambian businesses. Br Med I 1994;309: 1549-1550.

17. Jacquez JA, et al. Role of the primary infection in epidemics of HIV infection in gay cohorts. J Acquir Immune Defic Syndr 1994;7:1169-1184.

18. Grosskurth $\mathrm{H}$, et al. Impact of improved treatment of sexually transmitted diseases on HIV infection in rural Tanzania: randomised controlled trial. Lancet 1995;346:530-536.

19. Mertens T, et al. Prevention indicators to evaluate the progress of National AIDS Programmes. AIDS 1994;8:1359-1370.

20. Jain M, John T, Keusch G. A review of HIV infection in India. J Acquir Immune Defic Syndr 1994;7:1185-1194. 
ABSTRACT Routine surveillance of HIV (human immunodeficiency virus) infection and AIDS has been established over the past decade in many countries around the world. HIV estimates derived from empirical data are essential to the assessment of the HIV situation in different parts of the world and trends are used in tracking the development of regional epidemics, thereby keeping intervention activities focused on realities. As of the end of 1995, and following an extensive country-by-country review of HIV/AIDS data, a cumulative total of 6 million AIDS cases were estimated to have occurred in adults and children worldwide and currently 20.1 million adults are estimated to be alive and infected with HIV or have AIDS. Of the total prevalent HIV infections, the majority remain concentrated in eastern, central and southern Africa, but the epidemic is evolving with spread of infection from urban to rural areas, as well as to West and South Africa, India and Southeast Asia, and to a lesser extent-with proportional shifts to heterosexual infections-in North America, Western Europe and Latin America.

While the longer-term dimensions of the HIV epidemic at global level cannnot be forecast with confidence, WHO currently projects a cumulative total of close to 40 million HIV infections in men, women and children by the year 2000. By that time, the male:female ratio of new infections will be close to 1:1. Recent trends indicate that HIV prevalence levels may be stabilizing or even decreasing among pregnant women in southern Zaire and parts of Uganda, among military recruits aged 21 in Thailand, and in some populations of northern Europe and the USA. While these changes may take place as part of the intrinsic dynamic of the epidemic, there is some evidence that declines in HIV prevalence are related to declines in HIV incidence which are, at least partly, due to prevention efforts. The challenge of surveillance and evaluation methods is now to identify the ingredients of success which may reveal a glimmer of hope.

\section{Congreso de la Asociación Internacional de Odontología Pediátrica}

Fechas: $\quad 17$ al 20 de septiembre de 1997

Lugar: Buenos Aires, Argentina

La comisión organizadora del XVI Congreso de la Asociación Internacional de Odontología Pediátrica participa a todos los interesados que del 17 al 20 de septiembre de 1997 en el Marriott Plaza Hotel en Buenos Aires, Argentina, se llevará a cabo su congreso anual. Esta actividad internacional cuenta con el apoyo de la Asociación Argentina de Odontología para Niños, miembro de la Asociación Odontológica Argentina.

Información:

XVI Congreso de la Asociación Internacional de Odontología Pediátrica

Asociación Argentina de Odontología para Niños

Seccional AAON, Junín 959 (1113) Buenos Aires, Argentina

Teléfono (541) 961-6141

Fax (541) 961-1110 